\title{
Correlates of Depression among Patients Diagnosed with Chronic Illnesses in Saudi Arabia
}

\author{
Ahmad E. Aboshaiqah \\ College of Nursing, King Saud University, Riyadh, KSA \\ Email: aaboshaiqah@ksu.edu.sa
}

Received 6 June 2014; revised 21 July 2014; accepted 1 August 2014

Copyright (C) 2014 by author and Scientific Research Publishing Inc.

This work is licensed under the Creative Commons Attribution International License (CC BY). http://creativecommons.org/licenses/by/4.0/

(c) (i) Open Access

\begin{abstract}
Background: Depression contributes to an increased medical morbidity and mortality among patients with chronic medical illnesses. The purpose of this study was to investigate correlates of depression among patients diagnosed with chronic illnesses in Saudi Arabia. Methods: A cross sectional survey using 412 patients diagnosed with chronic illnesses has been used to collected data in regards to depressive symptoms, psychological distress, coping, and life satisfaction. Results: $\mathbf{2 5 . 2 \%}$ of the patients reported that they had moderate to severe depressive symptoms, and about $13.8 \%$ of them had mild level of depression. Psychological distress and life satisfaction were significant correlates with depressive symptoms $(r=0.33,0.54, p<0.001)$, while coping strategies is not. The results also showed that there is a significant and negative correlation between patients' age and depression score $(r=0.17, p \leq 0.001)$. Regarding gender differences, the analysis showed that there was no significant difference between male and female patients in their depressive symptoms $(t=-0.69, p=0.488)$. Conclusion: implication for clinical practice and research discussed.
\end{abstract}

\section{Keywords}

Chronic Illness, Depression, Stress, Life Satisfaction

\section{Introduction}

Chronic illness is becoming the most life devastating health problem and cause of death around the world. According to Yach and associates [1], cardiovascular diseases are the most leading cause of death around the world followed by cancer, chronic pulmonary diseases, and diabetes mellitus. Therefore, understanding the relation- 
ship between psychosocial wellbeing and chronic illnesses appears vital to public health assessment and health care delivery system. Health care policy makers are struggling to fulfill the requirement for patients with chronic illnesses given the increased patients' demands and cost of health care. During the last few decades, Saudi Arabia has witness a significant increase in number of deaths primarily due to chronic illness such as cardiovascular and cancer [2]. Thus, the comorbidity between chronic physical conditions and psychosocial health consequences such as depressive feelings, stress, adherence, and psychosocial health concerns became common interest for health professional and researchers. According to Doumit and Nasser [3], patients with chronic illnesses are overwhelmed with psychological stressors due to requirement related to management of their illnesses. However, patients' psychosocial status may interfere with their ability to manage their needs independently that may exacerbate their health condition [4]. For example, patients with chronic illnesses may suffer depressive feelings that delay their recovery and healing process [5]. Moreover, Frasure-Smith and Lespérance [6] found that one issue of concern among patients experiencing coronary atherosclerosis disease is the development of psychological problems such as anxiety, stress, and depression. Therefore, patients with chronic illnesses are struggling to manage their physical illnesses independently and further overwhelmed with vulnerability to increased psychosocial co-morbidity [4].

One of the significant psychosocial factors that influence patients' health conditions is depression. Number of studies connected depression to health treatment outcomes. For example, depression has been associated with increased medical morbidity, mortality, worse quality of life, risk for complications among patients with cardiac and metabolic problems [7] [8]. Furthermore, the literature showed that treatment of depression had positive outcome on patients' prognosis and quality of life, and that depressed mood lowers the force needed to cope with the chronic diseases, decreases tolerability of physical symptoms, and increases psychosocial disturbances [6]. Previous studies also showed that depression has been linked with chronic illnesses [9], and that patient' psychological difficulties and health care professional competency related to psychological follow up care have been linked to increased morbidity, mortality, and expenditure of health services [10]. According to Katon [11], depression contributed to $50 \%$ increase in health care cost medical illnesses.

The literature showed that the impact of chronic illnesses on the bio-psycho-social aspects of individual wellbeing is related to difficulties of individuals' adjustment to their illnesses [12]. Therefore, understanding the relationship between depression and other psychosocial factors such as coping, life satisfaction, and stress seem to be noteworthy in terms of understanding the factors that interfere with effectiveness of treatment plans. Previous studies showed that there is a relationship between difficulties in coping mechanisms and the development of depression among patients with chronic illnesses [13]. This infers that exploring factors that predict depression among patients with chronic illness will enable addressing the factors the possibly affecting patients' physical and psychological condition. Therefore, the purpose of this study is to examine the psychological and sociodemographic correlates of depression among patients with chronic illnesses in Saudi Arabia. The specific aims are:

- To identify the psychological and sociodemographic correlates of depression among patients with chronic illnesses in Saudi Arabia.

- To identify the differences depressive symptoms of patients with chronic illnesses in Saudi Arabia in relation to demographic and personal characteristics: age, gender, working status, and medical diagnosis.

\section{Methods}

\subsection{Design}

A quantitative approach using cross-sectional, descriptive-correlational design was used to examine correlates of depression among patients with chronic illness. Data was data from patients diagnosed with diabetes mellitus-type-II, Rheumatoid arthritis, CAD, cancer, and pulmonary diseases from two major tertiary hospitals in Saudi Arabia. Information collected in regards to depression, stress, and coping.

\subsection{Sample and Settings}

A convenience sampling of 412 completed and retuned the questionnaire. Sample size was estimated using computer program G. power 3.0 of correlation; normal bivariate model of small effect size of 0.15 , at alpha 0.05 two tailed level of significance, and power of 0.8 , to be at least 385. A total of 600 patients approached and invited to study participation and 412 completed and retuned the questionnaire with a response rate of $69 \%$. The 
study targeted patients attending primary, secondary and tertiary care units. Inclusion criteria include: 1) diagnosed with one of the following chronic illness longer than 6 months: diabetes mellitus-type-II, Rheumatoid arthritis, CAD, cancer, and pulmonary diseases; 2) age of 18 years or above; 3) ability to read and write in Arabic. Exclusion criteria included: no history of diagnosed mental or cognitive disorders.

\subsection{Data Collection Procedure}

Prior data collection, ethical approval obtained from the IRB at King Saud University, and the targeted institutions. Data are collected between November 2013 and January 2014. Data collected using self report format of data collection at patient's convenience. Patients who expressed interest to participation in the study were approached by the researcher who explained the study and provided them with all details and answered all their questions. Patients were asked to sign the consent form that included information related to the title of the study, its purpose, its significance and a statement informing the participants that their privacy would be protected by assuring them that their responses will be treated confidentially, and information that reveal their identity will not be recorded. Also, the information will be used for the purpose of the study, and that their participation is voluntary and they have the right to withdraw at any time during the study and that their decisions will not influence the quality of care they receive. Anonymity of the respondents ensured during and after study completion; and data secured and saved to provide anonymity. Interviews conducted by trained research assistants at private rooms or patients' rooms upon their convenience. Filling the survey required about 25 minutes and patients who need more time was given opportunity to take break and research assistants retuned to complete filling the questionnaire. The whole package presented in Arabic language.

\subsection{Instruments}

The data collected using an Arabic version of self-reporting questionnaires. The Instruments were:

1) The Beck Depression Inventory-II (BDI-II) [14] was used to assess patients' depressive symptoms, which contain items that measure cognitive-affective symptoms and attitudes, impaired performance, and somatic symptoms [14]. This instrument contains 21 questions answered on a four-point Likert scale in which 0 represents the absence of symptoms and 3 represents an extreme problem. The total range of 0 to 63 and standard cutoff points as follow: 0 - 13 indicates no or minimal symptom, 14 - 19 indicates mild symptoms, 20 - 28 indicates moderate symptoms, and 29 - 63 indicates severe symptoms [14]. A score of 13 is the cut-off point indicating depression. The test-retest $\mathrm{r}$ was 0.88 , and Cronbach’s Alpha is 0.87 [14]. In this study, Cronbach's Alpha was 0.79.

2) Stress was measured using the brief form of Psychological Stress Measure [15]. The original Psychological Stress Measure (PSM) was designed using 49 items drawn from descriptors generated by focus groups on stress. The scale is unifactorial in structure and maintains a test-retest stability of 0.68 to 0.80 under apparently constant conditions. Patients checks the answer that best indicates the degree to which each statement has applied to him/her recently The responses made on a Likert scale and ranged from range from 1 (null) to 4 (much). The higher the score in the scale reflect higher level of psychological stress. In this study, Cronbach's Alpha was 0.81 .

3) Coping skills was measured using the abbreviated version of the COPE Inventory [16]. Brief COPE is a 28 items scale measures the ways individuals use to cope with stress in their life. Brief COPE is formed of 14 domains (each consisted of 2 items) were responses ranged from 1 (I haven't been doing this at all) to 4 (I’ve been doing this a lot). The scale takes $>10$ minutes to be completed. The scale has good internal inconsistency with Cronbach's alpha of 0.83 [16]. In this study, Cronbach's Alpha was 0.73.

4) Life satisfaction was measured using the Satisfaction with Life Scale [17]. This is a general measure of life satisfaction, which consisted of five statements. Participants were asked to rate each statement according to the following seven-point scale: 1) strongly disagree, 2) disagree, 3) slightly disagree, 4) neither agree nor disagree, 5) slightly agree, 6) agree, and 7) strongly agree. The scores of the total scale ranges from 5 to 35 and interpreted as follow: from 31 - 35 (eextremely satisfied), from 26 - 30 (satisfied), from 21 - 25 (slightly satisfied), 20 (neutral), from 15 - 19 (slightly dissatisfied), from 10 - 14 (dissatisfied), and 5 - 9 (extremely dissatisfied). The test-retest reliability was estimated to be 0.87 [17]. In this study, Cronbach's Alpha was 0.76 .

Potential covariates: Gender, age, marital status, type of disease, duration of disease, smoking status, income, education level and work status. The demographic information obtained from an investigator-developed subject profile. 


\subsection{Data Analysis Plan}

The Statistical Package for Social Science (SPSS 18) software (IBM, Chicago, IL, USA) was considered as suitable software for data entry, storage and analysis. Descriptive statistical analysis such as frequency count, percentage, mean, median and standard deviation was employed to describe the research sample. The Pearson product moment correlation coefficient was used to describe the association between variables. T-test for two independent samples (or ANOVA as appropriate) was used to compare means. Statistical significance was set at $\mathrm{p}>0.05$.

\section{Results}

\subsection{Descriptive Characteristics}

A total number of 412 patients completed the questionnaire (see Table 1). Patients' age ranged from 18 to 98 years, with mean of $44.2(\mathrm{SD}=16.8)$. About $33.3 \%(n=138)$ of the patients there were male patients, while $66.6 \%(n=272)$ were females. In regard to marital status, the majority of them $28.7 \%(n=243)$ were married, while $5.3 \%(n=25)$ were divorced, and 20.8\% $(n=85)$ were single, and $12.8 \%(n=53)$ were widow. The analysis also showed that most of patients $(58.5 \%, n=242)$ were not working, and $21.0 \%(n=87)$ of them had a full time work, $13.0 \%(n=54)$ had retired, where the least percent $4.5 \%(n=19)$ of patients had a part time work.

In regard to their medical diagnosis, the analysis showed that $32.5 \%(n=134)$ of the patients had diabetes mellitus type-II, $17.5 \%(n=72)$ had cardiovascular disease, $15.5 \%(n=64)$ had pulmonary diseases, $19.8 \%(n=$ 81) had rheumatoid arthritis, and $14.7 \%(n=92)$ had cancer.

Table 1. Descriptive characteristics of patients diagnosed with chronic illnesses $(\mathrm{N}=412)$.

\begin{tabular}{|c|c|c|c|c|c|c|c|c|}
\hline \multicolumn{2}{|c|}{ Variable } & $\mathrm{n}$ & $\%$ & M & SD & Md. & $\mathrm{P}_{25}$ & $\mathrm{P}_{75}$ \\
\hline \multicolumn{2}{|c|}{ Age } & & & 44.2 & 16.8 & 45 & 29.5 & 55.3 \\
\hline \multirow{2}{*}{ Gender } & Male & 138 & 33.3 & & & & & \\
\hline & Female & 272 & 66.9 & & & & & \\
\hline \multirow{4}{*}{ Marital status } & Single & 86 & 20.8 & & & & & \\
\hline & Married & 243 & 28.7 & & & & & \\
\hline & Widow & 85 & 20.8 & & & & & \\
\hline & Divorced & 25 & 5.3 & & & & & \\
\hline \multirow{3}{*}{ Working status } & Not working & 242 & 58.2 & & & & & \\
\hline & Part-time job & 19 & 4.5 & & & & & \\
\hline & Full time job & 87 & 21.0 & & & & & \\
\hline \multirow{5}{*}{ Level of education } & $>$ High school & 169 & 40.3 & & & & & \\
\hline & High school & 104 & 25.1 & & & & & \\
\hline & Diploma & 32 & 7.7 & & & & & \\
\hline & Bachelor & 80 & 19.3 & & & & & \\
\hline & Graduate & 11 & 2.7 & & & & & \\
\hline \multirow{5}{*}{ Medical diagnoses } & Diabetes mellitus & 107 & 25.0 & & & & & \\
\hline & Cardiovascular & 105 & 25.4 & & & & & \\
\hline & Pulmonary & 50 & 12.1 & & & & & \\
\hline & Cancer & 68 & 15.9 & & & & & \\
\hline & Rheumatoid arthritis & 6 & 1.4 & & & & & \\
\hline
\end{tabular}

$\mathrm{P}_{25}$ : percentile $25^{\text {th }} ; \mathrm{P}_{75}$ : percentile $75^{\text {th }}$. 


\subsection{Psychosocial Health Factors}

Depression: Regarding depressive symptoms, the analysis (see Table 2) showed that the patients had a mean score of 14.7 ( $\mathrm{SD}=10.8$ ) with scores ranging from 0 to 55 . About $50 \%$ of the patients had a score of 13 or above. In regards to level of depression, the analysis showed that $41.1 \%(n=170)$ of the patients found to have no or minimal depressive symptoms, while $13.8 \%(n=57)$ had mild depressive symptoms, $6.8 \%(n=28)$ had moderate depressive symptoms, and $18.4 \%(n=83)$ had severe depressive symptoms. The analysis indicates that about $45 \%$ of the patients are suffering from moderate to severe depressive symptoms compared to $55 \%$ with no to mild depressive symptom.

Coping skills: Regarding patients' coping skills using brief COPE scale (see Table 2), the analysis showed that patients had a mean score of $7.2(\mathrm{SD}=12.5)$ with scores ranging from 28 to 110 . Considering that the possible range of score is $28-112$, and that the analysis showed that 50\% $(n=206)$ of the patients had a score of 72 or above and $50 \%$ of them had a score between 65 and 80 , the results indicate that patients, in general, had moderate ability to effectively cope with their life situations.

Life satisfaction: Regarding patients' satisfaction about their life (see Table 2), the analysis showed that patients had a mean score of 24.5 ( $\mathrm{SD}=6.3$ ) with scores ranging from 7 to 35 . Considering that the possible range of score is $5-35$, and that the analysis showed that $50 \%(n=206)$ of the patients had a score of 25 or above and $50 \%$ of them had a score between 20 and 29, the results indicate that patients, in general, had high level of satisfaction about their life.

Psychological distress: Regarding patients' psychological distress level (see Table 2), the analysis showed that patients had a mean score of 38.9 (SD = 11.9) with scores ranging from 12 to 70 . Considering that the possible range of score is $9-72$, and that the analysis showed that that $50 \%(n=206)$ of the patients had a score of 40 or above and $50 \%$ of them had a score between 31 and 47 , the results indicate that patients, in general, had moderate level of stress.

\subsection{Correlates and Differences in Depressive Symptoms in Relation to Demographic Characteristics}

Using Pearson correlation coefficient (r), the analysis showed that there is a positive and significant correlation between depression and stress $(\mathrm{r}=0.33, \mathrm{p}<0.001)$, and negative a significant correlation with life satisfaction $(-0.54, \mathrm{p}<0.001)$, while no statistical significant correlation found with coping $(\mathrm{r}=0.05, \mathrm{p}=0.39)$. This indicates that those who have higher level of stress and lower level of perception of life satisfaction are more likely to have higher level of depression. While, low correlation between coping and depression infers that depression among patients with chronic illness not dependent upon coping strategies used by the patients.

Regarding the relationship between demographic and personal characteristic and depressive symptoms, the analysis showed that there is a significant and negative correlation between patients' age and depression score ( $\mathrm{r}$ $=0.17, \mathrm{p} \leq 0.001)$. Regarding gender differences, the analysis showed that there was no significant difference between male and female patients in their depressive symptoms $(\mathrm{t}=-0.69, \mathrm{p}=0.488)$, although mean score of depression for female $(15.2, \mathrm{SD}=10.4)$ patients was higher than males $(14.3, \mathrm{SD}=11.5)$. To examine the differences in depression in relation to working status, one-way ANOVA was conducted. The analysis showed that there was no significant difference in depressive symptoms in regards to working status, $\left(\mathrm{F}_{3,325}=1.65, \mathrm{p}=\right.$ 0.147), and education level $\left(\mathrm{F}_{3,325}=1.95, \mathrm{p}=0.071\right)$. While there was a significant difference in depression level related to marital status $\left(\mathrm{F}_{3,325}=5.82, \mathrm{p}=0.001\right)$. Using post hoc comparison (Tukey HSD), the analysis showed

Table 2. Psychosocial health status of patients diagnosed with chronic illness ( $\mathrm{N}=412)$.

\begin{tabular}{cccccccccc}
\hline Variable & N & M & SD & Min & Max & $\mathbf{P}_{25}$ & $\mathbf{P}_{50}$ & $\mathbf{P}_{75}$ \\
\hline Depressive symptoms & 412 & 14.7 & 10.8 & 0.0 & 55 & 6.0 & 13.0 & 23.0 \\
Life satisfaction & 412 & 24.5 & 6.3 & 7.0 & 35 & 20.0 & 26.0 & 29.0 \\
Coping & 412 & 72.2 & 12.5 & 28.0 & 110. & 65.0 & 72.0 & 80.0 \\
Psychological distress & 412 & 39.0 & 11.6 & 12.0 & 70.0 & 31.0 & 40.0 & 47.0 \\
\hline
\end{tabular}

$\mathrm{P}_{25}$ : percentile $25^{\text {th }} ; \mathrm{P}_{50}$ : percentile $50^{\text {th }}$ (Median); $\mathrm{P}_{75}$ : percentile $75^{\text {th }}$. 
that single patients had the lowest mean score of depression $(\mathrm{M}=10.8)$ and single patients were the only group of patients whom are statistically significant (low) than all other groups.

Regarding differences in depressive symptoms in relation to patients' medical diagnoses (see Table 3), the analysis showed that more $24 \%$ and $21.2 \%$ of those diagnosed with diabetes mellitus and cancer have serve level of depression, while about $10 \%$ of the those who have more than one chronic illness suffer severe level of depression. Also the analysis showed that about $60 \%$ of those diagnosed with arthritis reported no or minimal depressive symptoms, however; $32 \%$ of them reported moderate to severe level of depression. It's apparent from the analysis that patients with more than one chronic illness have less depressive symptoms that those who have only one chronic illness. Using ANOVA test to examine the difference in mean score of depression among patients related to their chronic illness, the analysis showed that there were no significant differences $\left(\mathrm{F}_{5,325}=1.91\right.$, $\mathrm{p}=0.09$ ) although there differences in percentages across level of depression related to type of diagnosis.

\section{Discussion}

Globally, chronic physical health problems are the main cause for disability [18], and depression is associated with increased medical morbidity, mortality, and risk for complications among patients with chronic medical illnesses [7]. Depression also found to increase the health care cost even with suppression of physical severity [11]. Therefore; screening for psychological factors among patients diagnosed with chronic illnesses is considered a primary function for health professional caring for this group of patients [14] [15]. This study aimed at examining the correlates of depressive symptoms among patients diagnosed with chronic illnesses and explore further about the differences in depressive symptoms related to sociodemographic and medical conditions. The study found, in general, that a significant number of patients had moderate to severe depressive symptoms. In addition, the study showed that the depressive symptoms (moderate to severe) among the listed chronic illnesses ranged from 22\% (Pulmonarydisease) to 34\% (arthritis and cancer). The results of this study had some agreement with previous international reports. Globally, Moussavi and colleagues [19] found that prevalence of depressive episode among patients with chronic illnesses ranged from $2.0 \%$ (diabetes mellitus) to 4.5 (angina), and that the average depressive episode among those with more than one physical disorder ranged from $9.3 \%$ to 23.0\%. The prevalence of depressive symptoms in this study is higher than Moussavi and colleagues' report [19]. One explanation is that in this study a self report format of data has been used while in Moussavi and colleagues' reported data has been collected using interviews. Moreover, this study found that about $21.4 \%$ of patients with

Table 3. Difference in depression level among related to medical diagnosis $(\mathrm{N}=412)$.

\begin{tabular}{|c|c|c|c|c|c|c|c|}
\hline \multirow{2}{*}{\multicolumn{2}{|c|}{ Depression level }} & \multicolumn{6}{|c|}{ Medical diagnosis } \\
\hline & & Arthritis & Cancer & CVD & DM & Pulmonary disease & Comorbid \\
\hline \multirow{3}{*}{ None to minimal } & $\mathrm{n}$ & 4 & 22 & 59 & 34 & 21 & 30 \\
\hline & \% within depression level & 2.4 & 12.9 & 34.7 & 20.0 & 12.4 & 17.6 \\
\hline & \% within medical diagnosis & 66.7 & 39.3 & 65.6 & 40.5 & 53.8 & 53.6 \\
\hline \multirow{3}{*}{ Mild } & $\mathrm{n}$ & 0 & 12 & 8 & 17 & 7 & 13 \\
\hline & \% within depression level & 0 & 21.1 & 14.0 & 29.8 & 12.3 & 22.8 \\
\hline & \% within medical diagnosis & 0 & 21.4 & 8.9 & 20.2 & 17.9 & 23.2 \\
\hline \multirow{3}{*}{ Moderate } & $\mathrm{n}$ & 1 & 8 & 2 & 7 & 5 & 5 \\
\hline & \% within depression level & 3.6 & 28.6 & 7.1 & 25.0 & 17.9 & 17.9 \\
\hline & \% within medical diagnosis & 16.7 & 14.3 & 2.2 & 8.3 & 12.8 & 8.9 \\
\hline \multirow{3}{*}{ Severe } & $\mathrm{n}$ & 1 & 14 & 21 & 26 & 6 & 8 \\
\hline & \% within depression level & 1.3 & 18.4 & 27.6 & 34.2 & 7.9 & 10.5 \\
\hline & \% within medical diagnosis & 16.7 & 25.0 & 23.3 & 31.0 & 15.4 & 14.3 \\
\hline
\end{tabular}

CVD: Cardiovascular Disease; DM: Diabetes Mellitus. 
cancer had severe depressive symptoms, while in previous study the prevalence rates for depression in patients with cancer ranged from 22\% to 29\% [20] which is indicates that patients with cancer in Saudi Arabia had almost equal rates of depressive feeling compared to those in other regions in the world. Moreover, and similar to Zhang and associates [21], the majority of the patients in this study $(60 \%-70 \%)$ with pulmonary diseases, diabetes, CVD had depressive feelings. These rates were higher than what has been reported by previous studies that that $20 \%$ of patients with COPD had depressive feeling [20], and $51 \%$ of patients with congestive heart failure had mild to severe depressive symptoms on BDI [7]. In conclusion, significant number of patients with chronic illnesses suffers depressive feelings that may interfere negatively in their ability to adapt to their illness.

In regards to correlates of depression, we found that low perception of life satisfaction and psychological distress are associated depressive symptoms, whereas; coping, type of medical diagnoses, level of education and gender were not. Moreover, there was a significant difference between male and female patients in their level of depression. The results does not support previous reports that depression is associated with number of clinical and demographic characteristic of patients diagnosed with chronic illnesses [11]. Although literature shows connection between coping to depression among patients with chronic illness [6] [13], this study showed that depression and coping have weak correlations. This may question the role of coping mechanism in forming the psychological status of patients. One possible explanation is that patients had depressive feeling, however; they may have also utilized available sources of social support to manage the negative feeling resulting from depression. In conclusion, Saudi patients with chronic illness are suffering depression, and that improving their psychosocial health status and managing their stressful life events that enabled them to encounter their negative feelings that produce depression. One limitation for this study is that data were collected cross sectional, while a longitudinal one may allow better understanding for a cumulative experience over long period of time.

One limitation for this study is that data were cross sectional. A longitudinal study may allow better understanding for a cumulative experience over long period of time.

\section{Conclusion}

Managing comorbidity of physical and psychological problems in primary care is needed. Data from this study suggests that there is a higher incidence and prevalence of depression in patients with chronic medical illness. Moreover, the study suggest that managing stress, enhancing life satisfaction and coping are among the most influencing factors that encounter negative feeling and development of depression among patients with chronic illnesses. This study has an implication for psychosocial nurses and mental health professionals at the community and primary care settings. There is a need that psychosocial nurses and mental health professionals assess and screen for psychosocial factors: stress, depression, life satisfaction, and coping skills among patients with chronic illness in their routine checkups and visits to outpatients units. There also a need to develop large treatment trials aimed at improving outcomes of psychosocial wellbeing in medical illnesses to prospect the cost and burden of such illnesses. Future research must focus on establishing diagnostically reliable criteria measuring depression and other psychosocial factors.

\section{Acknowledgements}

The authors extend their appreciation to the College of Nursing Research Center and the Deanship of Scientific Research at King Saud University for funding this research.

\section{References}

[1] Yach, D., Hawkes, C., Gould, C.L. and Hofman, K.J. (2004) The Global Burden of Chronic Diseases: Overcoming Impediments to Prevention and Control. Journal of the American Medical Association, 291, 2616-2622. http://dx.doi.org/10.1001/jama.291.21.2616

[2] World Health Organization (2012) The Impact of Chronic Diseases in Saudi Arabia. Fact Sheet. http://www.who.int/chp/chronic_disease_report/en/

[3] Doumit, J. and Nasser, R. (2010) Quality of Life and Wellbeing of the Elderly in Lebanese Nursing Homes. International Journal of Health Care, 23, 72-93.

[4] Sareen, J., Cox, B.J., Clara, I. and Asmundson, G. (2005) The Relationship between Anxiety Disorders and Physical Disorders in the US: National Comorbidity Survey. Depression \& Anxiety, 21, 193-202.

http://dx.doi.org/10.1002/da.20072 
[5] Katon, W., Lin, E.H. and Kroenke, K. (2007) The Association of Depression and Anxiety with Medical Symptom Burden in Patients with Chronic Medical Illness. General Hospital Psychiatry, 29, 147-155. http://dx.doi.org/10.1016/j.genhosppsych.2006.11.005

[6] Frasure-Smith, N. and Lespérance, F. (2006) Recent Evidence Linking Coronary Heart Disease and Depression. The Canadian Journal of Psychiatry, 51, 730-715.

[7] Freedland, K.E., Rich, M.W., Skala, J.A., Carney, R.M., Dávila-Román, V.G. and Jaffe, A.S. (2003) Prevalence of Depression in Hospitalized Patients with Congestive Heart Failure. Psychosomatic Medicine, 65, 119-128. http://dx.doi.org/10.1097/01.PSY.0000038938.67401.85

[8] Lustman, P.J., Anderson, R.J., Freedland, K.E., de Groot, M., Carney, R.M. and Clouse, R.E. (2000) Depression and Poor Glycemic Control. Diabetes Care, 23, 934-942. http://dx.doi.org/10.2337/diacare.23.7.934

[9] Honyashiki, M., Ferri, C.P., Acosta, D., Guerra, M., Huang, Y., et al. (2011) Chronic Diseases among Older People and Co-Resident Psychological Morbidity: A 10/66 Dementia Research Group Population-Based Survey. International Psychogeriatric Association, 23, 1489-1501. http://dx.doi.org/10.1017/S1041610211000500

[10] Wang, P.S., Demler, O., Olfson, M., Pincus, H.A., Wells, K.B. and Kessler, R.C. (2006) Changing Profiles of Service Sectors Used for Mental Health Care in the United States. The American Journal of Psychiatry, 163, 1187-1198. http://dx.doi.org/10.1176/appi.ajp.163.7.1187

[11] Katon, W. (2003) Clinical and Health Services Relationships between Major Depression, Depressive Symptoms, and General Medical Illness. Biological Psychiatry, 54, 216-226. http://dx.doi.org/10.1016/S0006-3223(03)00273-7

[12] Chen, P.Y. and Chang, H.C. (2012) The Coping Process of Patients with Cancer. European Journal of Oncology Nursing, 16, 10-16. http://dx.doi.org/10.1016/j.ejon.2011.01.002

[13] Hamdan-Mansour, A., Halabi, J. and Dawani, H. (2009) Depression, Hostility, and Substance Use among University Students in Jordan. Mental Health and Substance Use: Dual Diagnosis, 2, 53-64. http://dx.doi.org/10.1080/17523280802593301

[14] Beck, G., Steer, R. and Brown, G. (1996) Manual for the Beck Depression Inventory-II. Psychological Corporation, San Antonio.

[15] Lemyre, L., Tessier, R. and Eillion, L. (1990) Mesure du stress psychotogique (MSP): Manuel d'utilisation (Psychological Stress Measure). Editions Behaviora, Brossard.

[16] Carver, C.S. (1990) You Want to Measure Coping but Your Protocol's Too Long: Consider the Brief COPE. International Journal of Behavioral Medicine, 4, 92-100. http://dx.doi.org/10.1207/s15327558ijbm0401_6

[17] Diener, E., Emmons, R.A., Larsen, R.J. and Griffin, S. (1985) The Satisfaction with Life Scale. Journal of Personality Assessment, 49, 71-75. http://dx.doi.org/10.1207/s15327752jpa4901_13

[18] Mathers, C.D. and Loncar, D. (2005) Updated Projections of Global Mortality and Burden of Disease 2002e2030: Data Sources, Methods and Results. World Health Organization, Geneva.

[19] Moussavi, S., Chatterji, S., Verdes, E., Tandon, A., Patel, A. and Ustun, B. (2007) Depression, Chronic Diseases, and Decrements in Health: Results from the World Health Surveys. Lancet, 370, 851-858. http://dx.doi.org/10.1016/S0140-6736(07)61415-9

[20] Hotopf, M., Chidgey, J., Addington-Hall, J. and Ly, K.L. (2002) Depression in Advanced Disease: A Systematic Review, Part 1. Prevalence and Case Finding. Palliative Medicine, 16, 81-97. http://dx.doi.org/10.1191/02169216302pm507oa

[21] Di Marcoa, F., Vergaa, M., Reggentea, M., Casanovaa, F.M., Santusa, P., Blasib, F., Allegrab, L. and Centannia, S. (2006) Anxiety and Depression in COPD Patients: The Roles of Gender and Disease Severity. Respiratory Medicine, 100, 1767-1774. http://dx.doi.org/10.1016/j.rmed.2006.01.026 
Scientific Research Publishing (SCIRP) is one of the largest Open Access journal publishers. It is currently publishing more than 200 open access, online, peer-reviewed journals covering a wide range of academic disciplines. SCIRP serves the worldwide academic communities and contributes to the progress and application of science with its publication.

Other selected journals from SCIRP are listed as below. Submit your manuscript to us via either submit@scirp.org or Online Submission Portal.
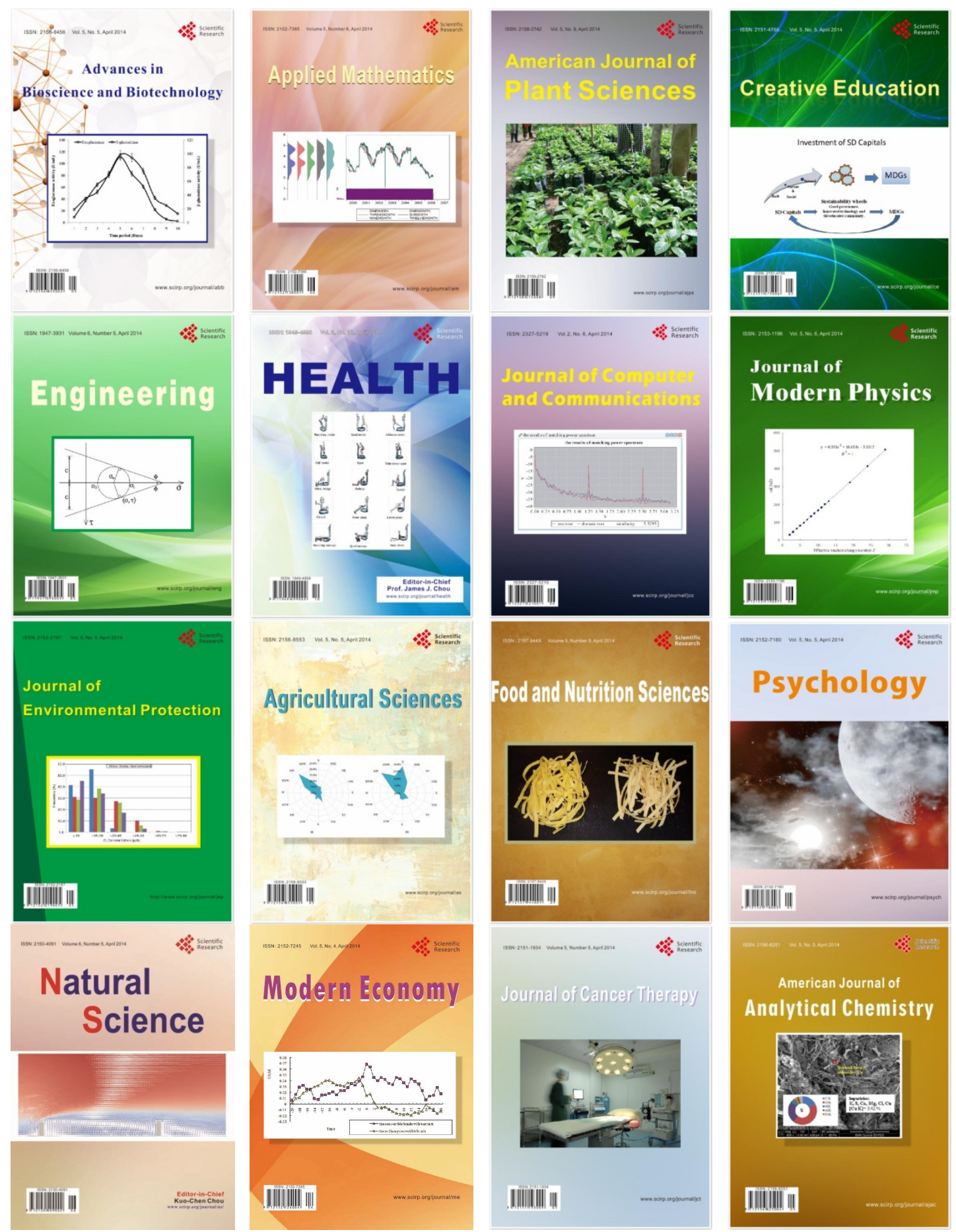